\title{
PECS II Block for Proximal Upper Limb Fistula Surgery
}

\section{Geraldine Cheong PC ${ }^{1}$, Jessica Teng SX${ }^{1}$, Ooi Desmond $\mathrm{GS}^{2}$, Simon Tan YS ${ }^{1}$, Wong $\mathrm{MH}^{1}$ ${ }^{1}$ Department of Anaesthesia, ${ }^{2}$ Department of Surgery, Khoo Teck Puat Hospital, Singapore}

\section{Background}

Upper limb fistula surgeries are often performed under brachial plexus blocks (BPB). However, the proximal site surgical incision close to the axilla frequently extends beyond coverage by a BPB and requires either rescue local anaesthetic (LA) supplementation or general anaesthesia. The PECS block, originally done for breast surgery, incorporates a PECS II component which covers the axilla and upper arm. The PECS II block is done by depositing LA in between the pectoralis minor and the serratus anterior muscle.

\section{Case Presentation}

We report a patient presenting for left brachio-basilic arterio-venous graft creation surgery done successfully under a left supraclavicular and left PECS II block. He is a 75-year old gentleman with hypertension, hyperlipidemia and end-stage renal failure requiring haemodialysis .

A left supraclavicular BPB (Figure 1) and PECS II block (Figure 2) were done under ultrasound guidance with Ropivacaine $0.4 \% 20 \mathrm{~mL}$ and Ropivacaine $0.3 \% 20 \mathrm{~mL}$ respectively with a BBraun Stimuplex $50 \mathrm{~mm} \mathrm{22G}$ insulated needle.
The surgery involved two incisions - above the elbow crease and just distal to the axilla (Figure 3). After the brachial artery and basilic vein were dissected, a vascular graft was tunneled subcutaneously in the anterior arm. Surgical duration was 2 hours. The patient remained pain free intraoperatively with no supplemental LA. The surgery was completed uneventfully. He was discharged on the same day and the numbness resolved within 24 hours.

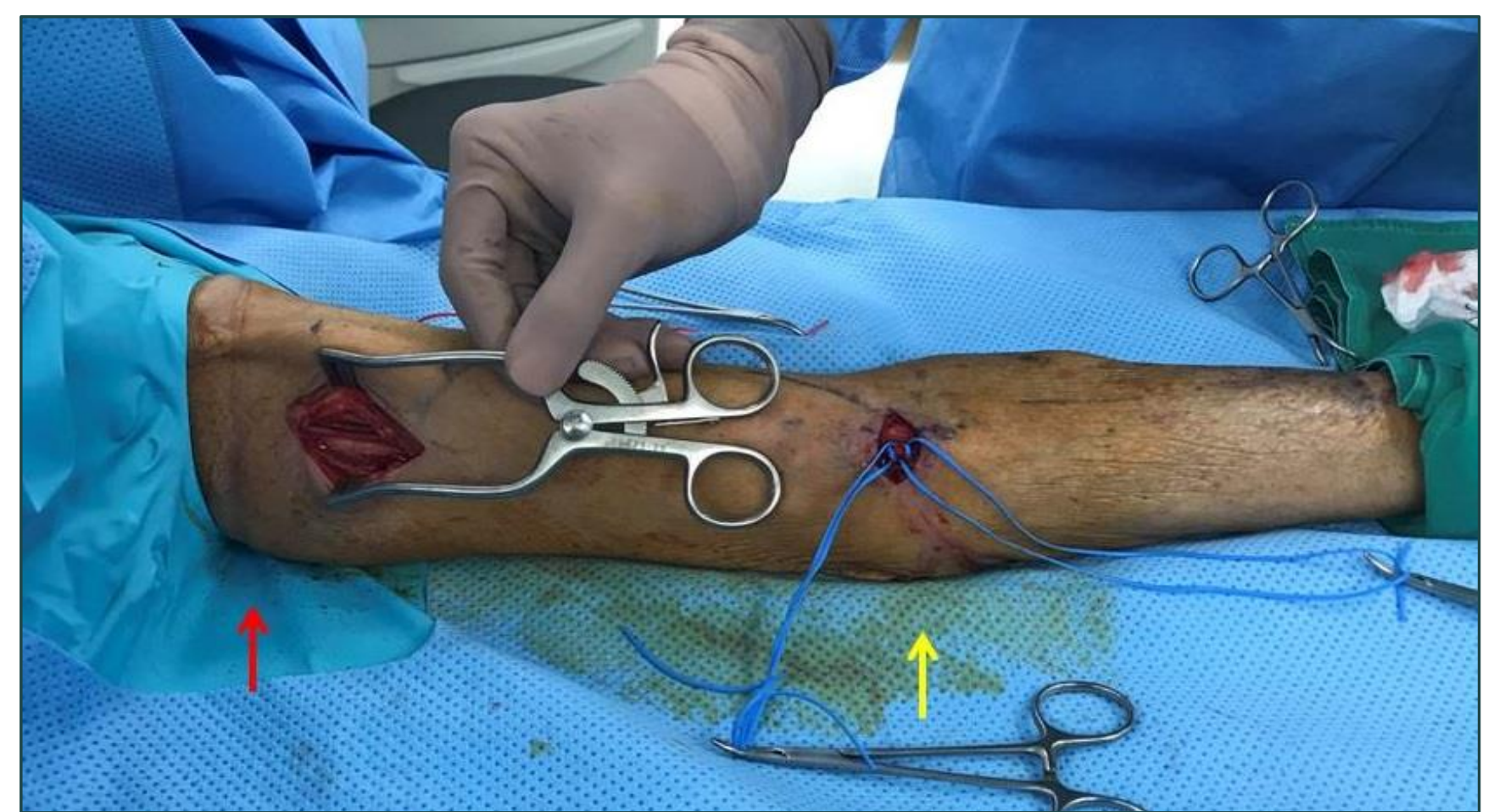

Figure 3. Arteriovenous graft surgical incisions at the cubital fossa (yellow arrow) and just distal to the axilla (red arrow).

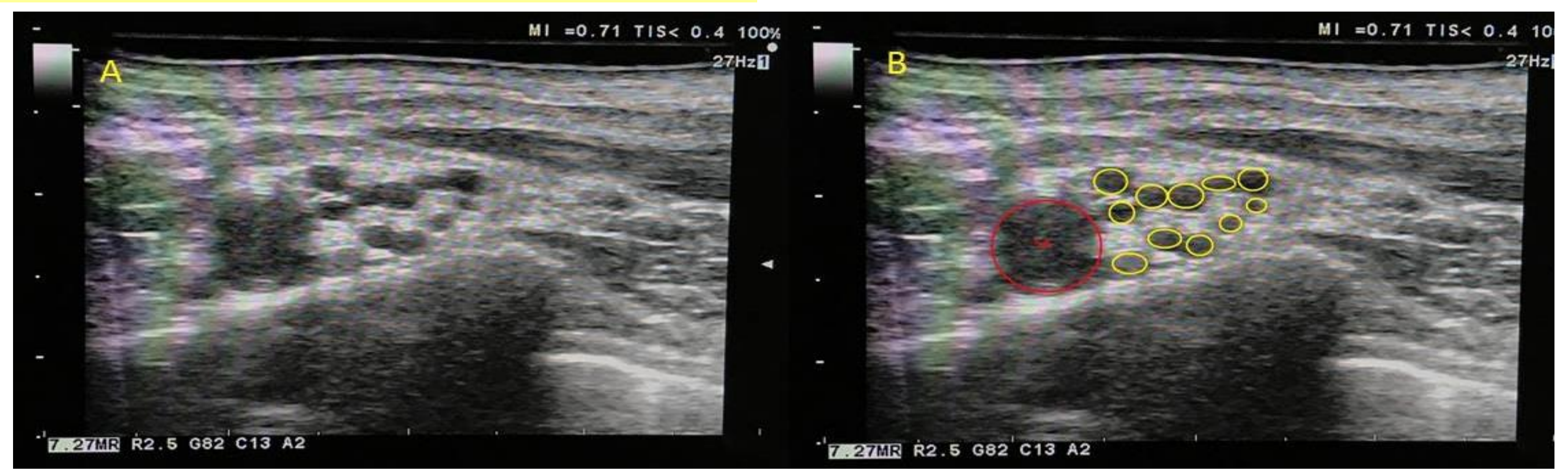

Figure 1A) Ultrasound image obtained during supraclavicular brachial plexus block. B) Annotated image. (SA = subclavian artery. Yellow circles $=$ brachial plexus)

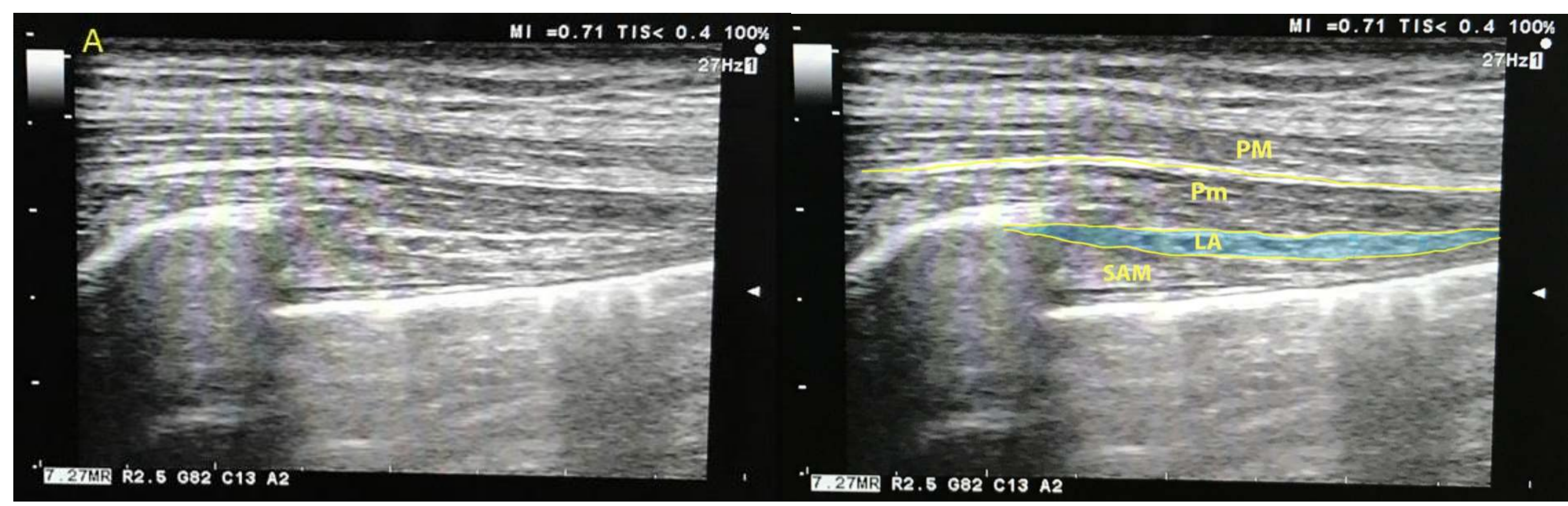

Figure 2 A) Ultrasound image of PECS II block. B) Same image annotated. (PM = Pectoralis major, $\mathrm{Pm}=$ pectoralis minor, $\mathrm{LA}=$ Local anaesthetic deposit, SAM = serratus anterior muscle)

Conclusion

Addition of the PECS II block to a BPB for proximal upper limb fistula surgeries can be considered to avoid the need for LA supplementation by the surgeon or conversion to GA in these potential high-risk patients. Further studies can be considered to investigate its potential benefit. 